Bull. Austral. Math. Soc.

VOL. 56 (1997) [149-156]

\title{
ON THE IMPLICIT DARBOUX PROBLEM IN BANACH SPACES
}

\section{DARIA WóJTOWICZ}

In this paper we prove the existence theorem for the implicit Darboux problem on the quarterplane $x \geqslant 0, y \geqslant 0$. Moreover, we study the topological structure of the solution set of this problem.

\section{InTRODUCTION}

In this paper we shall consider the following implicit Darboux problem

$$
\begin{aligned}
\frac{\partial^{2} z}{\partial x \partial y} & =g\left(x, y, z, \frac{\partial^{2} z}{\partial x \partial y}\right), \\
z(x, 0) & =0, \quad 0 \leqslant x<+\infty, \\
z(0, y) & =0, \quad 0 \leqslant y<+\infty,
\end{aligned}
$$

in a Banach space, where $\frac{\partial^{2} z}{\partial x \partial y}$ denotes the mixed derivative of $z$. We shall give sufficient conditions for the existence of a solution of (1). Moreover, under the same assumptions we shall prove an Aronszajn type theorem for this problem.

In our considerations we shall apply the following two theorems.

THEOREM 1 [3] Let $D$ be a closed and convex subset of a Hausdorff locally convex space such that $0 \in D$, and let $G$ be a continuous mapping of $D$ into itself. If the implication

$$
(V=\operatorname{conv} G(V) \text { or } \quad V=G(V) \cup\{0\}) \Longrightarrow V \text { is relatively compact }
$$

holds for every subset $V$ of $D$, then $G$ has a fixed point.

THEOREM 2 [7] Let $X, Y$ be metric spaces. Assume that $y$ is a point of $Y$ with a neighbourhood homeomorphic to a closed convex subset of a Fréchet space. Let $T: X \rightarrow$ $Y$ be a continuous $y$-closed mapping, and $T_{n}: X \rightarrow Y$ a homeomorphism into. If $y$ is an interior point of $\bigcap_{n=1}^{\infty} T_{n}(X)$ and $T^{-1}(y)$ is compact and nonempty, then $T^{-1}(y)$ is an $R_{\delta}$

Received 30th September, 1996

I would like to thank to dr D. Bugajewski for his valuable suggestions.

Copyright Clearance Centre, Inc. Serial-fee code: 0004-9729/97 \$A2.00+0.00. 
whenever $\lim _{n \rightarrow \infty} T_{n}=T$ uniformly on $T^{-1}(y)$ and all sets of the form $\bigcup_{n=1}^{\infty} T_{n}^{-1}(C)$, where $C$ is a compact subset of $\bigcap_{n=1}^{\infty} T_{n}(X)$.

Recall that a subset of a metric space is an $R_{\delta}$ if it is homeomorphic to the intersection of a decreasing sequence of compact absolute retracts.

Our main condition that guarantee the existence of the solution of (1) will be formulated in terms of the measure of noncompactness $\alpha$ introduced by Kuratowski (see [2] for the definition and basic properties).

\section{AN EXISTENCE THEOREM}

Let $I=[0,+\infty)$ and let $E$ be a Banach space. Assume that:

$1^{0} g: I \times I \times E \times E \rightarrow E$ is a continuous mapping;

$2^{0}$ there exists a number $k \in[0,1)$ such that

$$
\left\|g\left(x, y, u, v_{1}\right)-g\left(x, y, u, v_{2}\right)\right\| \leqslant k\left\|v_{1}-v_{2}\right\|
$$

for every $(x, y, u) \in I \times I \times E$ and $v_{1}, v_{2} \in E$;

$3^{0}$ for every $a, b>0$ there exists $m(a, b) \in \mathbb{R}_{+}$such that

$$
\|g(x, y, u, 0)\| \leqslant m(a, b) \quad \text { whenever } \quad|x|<a,|y|<b .
$$

First we shall show that (1) is equivalent to some Darboux problem in the explicit form. Indeed, consider the sequence of functions $f_{n}: I \times I \times E \rightarrow E$ such that $f_{0}(x, y, u)=$ $0, f_{n+1}(x, y, u)=g\left(x, y, u, f_{n}(x, y, u)\right)$ for every $(x, y, u) \in I \times I \times E$ and $n \in\{0,1,2, \ldots\}$. By $2^{0}$, in view of the Banach contraction principle, for every $(x, y, u) \in I \times I \times E$ there exists exactly one element $f(x, y, u) \in E$ such that $f(x, y, u)=g(x, y, u, f(x, y, u))$ and $f(x, y, u)=\lim _{n \rightarrow \infty} f_{n}(x, y, u)$. Hence the mapping $(x, y, u) \rightarrow f(x, y, u)$ satisfies the equation

$$
f(x, y, u)=g(x, y, u, f(x, y, u)) .
$$

Moreover, for every $n, p \in \mathbb{N}$ we have

$$
\left\|f_{n+p}(x, y, u)-f_{n}(x, y, u)\right\| \leqslant \frac{k^{n}}{1-k} m(a, b),
$$

whenever $|x|<a,|y|<b$. Thus $f_{n} \rightarrow f$ as $n \rightarrow \infty$, uniformly on every bounded subset of $I \times I \times E$. Hence the mapping $f: I \times I \times E \rightarrow E$ is continuous and

$$
\|f(x, y, u)\| \leqslant M(a, b) \text { for }|x|<a,|y|<b,
$$

where $M(a, b)=1 /(1-k) m(a, b)$. 
We note that the mapping $z: I \times I \rightarrow E$ is a solution of (1) if and only if it is a solution of the following Darboux problem

$$
\begin{aligned}
\frac{\partial^{2} z}{\partial x \partial y} & =f(x, y, z), \\
z(x, 0) & =0, \quad 0 \leqslant x<+\infty, \\
z(0, y) & =0, \quad 0 \leqslant y<+\infty .
\end{aligned}
$$

Now, we shall prove the following

LEMma Let $h: \mathbb{R}_{+} \rightarrow \mathbb{R}_{+}$be a function such that

$$
\alpha(g(A \times X \times Y)) \leqslant \max (h(\alpha(X)), \alpha(Y))
$$

for all bounded subsets $A \subset I \times I$ and $X \times Y \subset E \times E$. Then

$$
\alpha(f(A \times Z)) \leqslant h(\alpha(Z))
$$

for all bounded subsets $A \subset I \times I$ and $Z \subset E$ (see [5]).

Proof: From the definition of the sequence $\left(f_{n}\right)$ and (4), by mathematical induction we have

$$
\alpha\left(f_{n}(A \times Z)\right) \leqslant h(\alpha(Z))
$$

for all bounded subsets $A \subset I \times I$ and $Z \subset E$.

Fix $\varepsilon>0$. Since $f_{n} \rightarrow f$ uniformly on every bounded subset of $I \times I \times E$, as $n \rightarrow \infty$,

$$
f(A \times Z) \subset f_{n}(A \times Z)+K(0, \varepsilon)
$$

for all bounded subsets $A \subset I \times I$ and $Z \subset E$, and for sufficiently large $n \in \mathbb{N}$, where $K(0, \varepsilon)$ denotes the open ball of center 0 and radius $\varepsilon$ in $E$.

Hence

$$
\alpha(f(A \times Z)) \leqslant \alpha\left(f_{n}(A \times Z)\right)+2 \varepsilon \leqslant h(\alpha(Z))+2 \varepsilon .
$$

Since $\varepsilon>0$ is arbitrary, we receive (4).

Our first main result is given by the following

Theorem 3 If $g$ satisfies $1^{0}-3^{0}$ and (4), where $h: \mathbb{R}_{+} \rightarrow \mathbb{R}_{+}$is a continuous, nondecreasing function such that the inequality

$$
0 \leqslant u(x, y) \leqslant \int_{0}^{x} \int_{0}^{y} h(u(t, s)) d t d s, \quad(x, y) \in I \times I
$$

has only a trivial solution, then the problem (1) has a solution.

The above theorem extends the main result from [6]. 
Proof: Let $C=C(I \times I, E)$ be the space of all continuous functions $I \times I \rightarrow E$ with the topology of uniform convergence on each compact subset of $I \times I$. Set

$$
F(z)(x, y)=\int_{D(x, y)} f(t, s, z(t, s)) d t d s,
$$

$(x, y) \in I \times I, z \in C$ and $D(x, y)=\{(t, s) \in I \times I: 0 \leqslant t \leqslant x, 0 \leqslant s \leqslant y\}$. Obviously the operator $F$ maps $C$ into $C$ and is continuous. Let $D=\overline{\operatorname{conv}}(F(C) \cup\{0\})$. It is clear that $F$ maps $D$ into itself. We shall show now that $F$ satisfies (2).

Indeed, let $V$ be a subset of $D$ such that $V \subset \overline{\operatorname{conv}}(F(V) \cup\{0\})$. First, we verify that $V$ is equicontinuous on every compact subset of $I \times I$. Since

$$
\begin{aligned}
\left\|F(z)\left(x_{1}, y_{1}\right)-F(z)\left(x_{2}, y_{2}\right)\right\| & =\left\|\int_{D\left(x_{1}, y_{1}\right)} f(t, s, z(t, s)) d t d s-\int_{D\left(x_{2}, y_{2}\right)} f(t, s, z(t, s)) d t d s\right\| \\
& \leqslant \mu\left(D\left(x_{1}, y_{1}\right)-D\left(x_{2}, y_{2}\right)\right) M(a, b)
\end{aligned}
$$

where $\left|x_{1}\right|<a,\left|x_{2}\right|<a,\left|y_{1}\right|<b,\left|y_{2}\right|<b, z \in C$ the family $F(C)$ is equicontinuous on every compact subset of $I \times I$. Hence $V$ is equicontinuous on every compact subset of $I \times I$.

Let $W=F(V), v(x, y)=\alpha(V(x, y))$ and $w(x, y)=\alpha(W(x, y))$ for $(x, y) \in I \times I$. From the basic properties of $\alpha$ we obtain

$$
\begin{aligned}
v(x, y) & =\alpha(V(x, y)) \leqslant \alpha(\overline{\operatorname{conv}}(F(V)(x, y) \cup\{0\})) \\
& =\alpha(F(V)(x, y) \cup\{0\})=\max (\alpha(F(V)(x, y)), \alpha(\{0\})) \\
& =\alpha(F(V)(x, y))=w(x, y), \quad(x, y) \in I \times I
\end{aligned}
$$

and, similarly,

$$
\alpha(V(T)) \leqslant \alpha(W(T)) \quad \text { for each compact subset } \quad T \text { for } I \times I .
$$

Further, we have

$$
\begin{aligned}
& \left|w\left(x_{1}, y_{1}\right)-w\left(x_{2}, y_{2}\right)\right|=\left|\alpha\left(W\left(x_{1}, y_{1}\right)\right)-\alpha\left(W\left(x_{2}, y_{2}\right)\right)\right| \\
& \quad=\left|\alpha\left(F(V)\left(x_{1}, y_{1}\right)\right)-\alpha\left(F(V)\left(x_{2}, y_{2}\right)\right)\right| \\
& \quad \leqslant \sup _{u, v \in V}\left\|F(u)\left(x_{1}, y_{1}\right)-F(u)\left(x_{2}, y_{2}\right)-F(v)\left(x_{1}, y_{1}\right)+F(v)\left(x_{2}, y_{2}\right)\right\| \\
& \quad \leqslant 2 \sup _{u, v \in V}\left\|F(u)\left(x_{1}, y_{1}\right)-F(u)\left(x_{2}, y_{2}\right)\right\|, \quad\left(x_{1}, y_{1}\right),\left(x_{2}, y_{2}\right) \in I \times I .
\end{aligned}
$$

By the above inequality and the equicontinuity $F(V)$ on every compact subset of $I \times I$, we deduce that $w$ is continuous on every compact subset of $I \times I$. Hence $w$ is continuous on $I \times I$.

Divide the rectangle $D(x, y)$ into $n^{2}$ parts: $0=x_{0}<x_{1}<\ldots<x_{n}=x, 0=$ $y_{0}<y_{1}<\ldots<y_{n}=y$ in such a way that $x_{i}-x_{i-1}<1 / n$ and $y_{j}-y_{j-1}<1 / n$ 
for $i, j=1, \ldots, n$. Put $D_{i j}(x, y)=\left[x_{i-1}, x_{i}\right] \times\left[y_{j-1}, y_{j}\right], i, j=1, \ldots, n$. Since $W$ is equicontinuous and uniformly bounded on every compact subset of $I \times I$, by Ambrosetti's Lemma [1] and the continuity of $w$ there exists $\left(p_{i}, q_{j}\right) \in D_{i j}(x, y)$ such that

$$
\begin{aligned}
\alpha\left(W\left(D_{i j}(x, y)\right)\right) & =\sup _{(\boldsymbol{t}, \mathbf{s}) \in D_{\mathbf{i} j}(x, y)} \alpha(W(t, s))=\sup _{(t, s) \in D_{\mathbf{i} j}(x, y)} w(t, s) \\
& =w\left(p_{i}, q_{j}\right), \quad(x, y) \in I \times I .
\end{aligned}
$$

From the mean value theorem, for every $z \in V$ we obtain

$$
\begin{aligned}
F(z)(x, y) & =\int_{D(x, y)} f(t, s, z(t, s)) d t d s \\
& =\sum_{i, j=1}^{n} \int_{D_{i j}(x, y)} f(t, s, z(t, s)) d t d s \\
& \in \sum_{i, j=1}^{n} \mu\left(D_{i j}(x, y)\right) \overline{\operatorname{conv}} f\left(D_{i j}(x, y) \times z\left(D_{i j}(x, y)\right)\right), \quad(x, y) \in I \times I .
\end{aligned}
$$

Thus

$$
F(V)(x, y) \subset \sum_{i, j=1}^{n} \mu\left(D_{i j}(x, y)\right) \overline{\operatorname{conv}} f\left(D_{i j}(x, y) \times V\left(D_{i j}(x, y)\right)\right),
$$

$(x, y) \in I \times I$.

Hence, by the Lemma, the properties of $\alpha,(8)$ and (7) we have

$$
\begin{aligned}
w(x, y) & \leqslant \sum_{i, j=1}^{n} \mu\left(D_{i j}(x, y)\right) \alpha\left(f\left(D_{i j}(x, y)\right) \times V\left(D_{i j}(x, y)\right)\right) \\
& \leqslant \sum_{i, j=1}^{n} \mu\left(D_{i j}(x, y)\right) h\left(\alpha\left(V\left(D_{i j}(x, y)\right)\right)\right) \\
& \leqslant \sum_{i, j=1}^{n} \mu\left(D_{i j}(x, y)\right) h\left(\alpha\left(W\left(D_{i j}(x, y)\right)\right)\right) \\
& =\sum_{i, j=1}^{n} \mu\left(D_{i j}(x, y)\right) h\left(w\left(p_{i}, q_{j}\right)\right) \quad(x, y) \in I \times I .
\end{aligned}
$$

If $n \rightarrow \infty$, by the continuity of $h$ and $w$ we obtain

$$
w(x, y) \leqslant \int_{D(x, y)} h(w(t, s)) d t d s, \quad(x, y) \in I \times I .
$$

Thus, by (6) $w(x, y)=0$ and, therefore by (7), $v(x, y)=0$ for every $(x, y) \in I \times I$. Hence $V(x, y)$ is relatively compact for every $(x, y) \in I \times I$. In view of the generalisation of Ascoli's Theorem $[4, \mathrm{p} .81], V$ is relatively compact.

The operator $F$ satisfies all the assumptions of Theorem 1 and, therefore, there exists $z \in D$ such that $z=F(z)$. This completes the proof of Theorem 3 . 


\section{An ARONSZAJN PROPERTY}

The aim of this Section is to prove the following Aronszajn type theorem.

THEOREM 4 Under the assumptions of Theorem 3 the set $S$ of all solutions of (1) on $I \times I$ is an $R_{\delta}$.

ProOF: Let $F: C \rightarrow C$ be the operator defined in the proof of Theorem 3 and let $T=I-F$, where $I$ denotes the identity map. Obviously $T$ is continuous mapping of $C$ into itself. Now, we verify that $T$ is 0 -closed, that is, the following implication

$$
0 \in \overline{T(V)} \Longrightarrow 0 \in T(V)
$$

holds for every closed subset $V \subset C$. It is enough to verify that $T$ is a proper map, that is, if $Z$ is relatively compact, then $T^{-1}(Z)$ is relatively compact.

Let $Z \subset C$ be a relatively compact set and put $U=T^{-1}(Z)$. Consider the sequence $\left(u_{n}\right)$, where $u_{n} \in U$ for $n \in \mathbb{N}$. Set $V=\left\{u_{n}: n \in \mathbb{N}\right\}$. Since $V(x, y) \subset$ $(I-F)(V)(x, y)+F(V)(x, y) \subset \bar{Z}(x, y)+F(V)(x, y), \alpha(V(x, y)) \leqslant \alpha(\bar{Z}(x, y))+$ $\alpha(F(V)(x, y))=\alpha(F(V)(x, y)),(x, y) \in I \times I$.

By arguing similarly as in the proof of Theorem 3, we infer that $V$ is relatively compact. Hence there exists a convergent subsequence $\left(u_{n_{k}}\right)$ of $\left(u_{n}\right)$, so $U$ is relatively compact.

Define

$$
F_{n}(z)(x, y)=\int_{D\left(r_{n}(x, y)\right)} f(t, s, z(t, s)) d t d s, \quad(x, y) \in I \times I, z \in C, n \in \mathbb{N},
$$

where

$$
r_{n}(x, y)=\left\{\begin{array}{cc}
0, & (x, y) \in K(1 / n), \\
(1-1 /(\|(x, y)\|) n)(x, y), & (x, y) \in(I \times I) \backslash K(1 / n),
\end{array}\right.
$$

$K(1 / n)=\left\{(x, y): x \geqslant 0, y \geqslant 0, \sqrt{x^{2}+y^{2}} \leqslant 1 / n\right\}$.

Obviously, the operators $F_{n}$ map $C$ into itself and are continuous. Put $T_{n}=I-F_{n}$, $n \in \mathbb{N}$. Now, we shall prove that $T_{n}$ is a homeomorphism of $C$ into itself for every $n \in \mathbb{N}$. Obviously the mappings $T_{n}$ are continuous. Fix $n \in \mathbb{N}$. It is easy to see that for any $z_{1}, z_{2} \in C$

$$
T_{n}\left(z_{1}\right)=T_{n}\left(z_{2}\right) \Longrightarrow z_{1}=z_{2}
$$

It is enough to prove the continuity of $T_{n}^{-1}$. Assume that $z_{i}, z_{0} \in C, T_{n}\left(z_{i}\right) \rightarrow T_{n}\left(z_{0}\right)$, as $i \rightarrow \infty$. We have $F_{n}\left(z_{i}\right)(x, y)=F_{n}\left(z_{0}\right)(x, y)=0$ for $(x, y) \in K(1 / n)$, so $z_{i} \rightarrow z_{0}$ uniformly on $K(1 / n)$, as $i \rightarrow \infty$. Since $f\left(t, s, z_{i}(t, s)\right) \rightarrow f\left(t, s, z_{0}(t, s)\right)$ uniformly on $K(1 / n)$, as $i \rightarrow \infty$,

$$
\int_{D\left(r_{n}(x, y)\right)} f\left(t, s, z_{i}(t, s)\right) d t d s \rightarrow \int_{D\left(r_{n}(x, y)\right)} f\left(t, s, z_{0}(t, s)\right) d t d s
$$




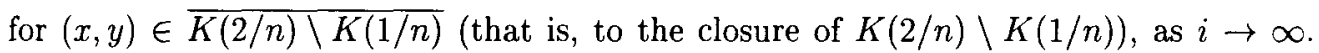
Hence, it is clear that $z_{i} \rightarrow z_{0}$ uniformly on $\overline{K(2 / n) \backslash K(1 / n)}$. By arguing similarly to the above, we infer that $z_{i} \rightarrow z_{0}$ uniformly on every compact subset of $I \times I$, as $i \rightarrow \infty$. This proves the continuity of $T_{n}^{-1}$.

Now, we shall show that $\lim _{n \rightarrow \infty} T_{n}=T$ uniformly. Fix a set $K(r), r>0$. Choose $n \in \mathbb{N}$ such that $K(1 / n) \subset K(r)$. From the inequalities

$$
\begin{aligned}
\left\|F_{n}(z)(x, y)-F(z)(x, y)\right\| & =\left\|\int_{D(x, y)} f(t, s, z(t, s)) d t d s\right\| \\
& \leqslant M\left(\frac{1}{n}, \frac{1}{n}\right) \frac{1}{n^{2}}, \quad \text { for }(x, y) \in K\left(\frac{1}{n}\right), z \in C,
\end{aligned}
$$

and

$$
\begin{aligned}
& \left\|F_{n}(z)(x, y)-F(z)(x, y)\right\| \\
& \quad=\left\|\int_{D\left(r_{n}(x, y)\right)} f(t, s, z(t, s)) d t d s-\int_{D(x, y)} f(t, s, z(t, s)) d t d s\right\| \\
& \quad \leqslant \frac{1}{n}\left(2 r-\frac{1}{n}\right) M(r, r) \text { for }(x, y) \in K(r) \backslash K\left(\frac{1}{n}\right), z \in C,
\end{aligned}
$$

it is clear that $F_{n}(z) \rightarrow F(z)$ uniformly in $z$, on every compact subset of $I \times I$.

Further, since $T^{-1}(0)$ is the set of all fixed points of $F$, by Theorem 3 it is nonempty. Let $\left(z_{k}\right)$ be sequence such that $z_{k} \in T^{-1}(0)$ for $k \in \mathbb{N}$. Put $V=\left\{z_{k}: k \in \mathbb{N}\right\}$. Obviously $V=F(V)$. By arguing similarly as in the proof of Theorem 3, we deduce that $V$ is relatively compact. Hence $T^{-1}(0)$ is relatively compact. Since it is closed, it is compact.

To complete our proof, it is enough to show that 0 is an interior point of $\bigcap_{n=1}^{\infty} T_{n}(C)$. We shall prove that $C \subset\left(I-F_{k}\right)(C)$ for every $k \in \mathbb{N}$. Fix $k \in \mathbb{N}$ and $z \in C$. Define a sequence $\left(u_{i}\right), u_{i} \in C$ in the following way:

$$
\begin{aligned}
& u_{1}(x, y)=z(x, y), \quad(x, y) \in K\left(\frac{1}{k}\right) \\
& \widetilde{u_{i}}(x, y) \text { is a continuous extension of } u_{i}(x, y) \text { from } K\left(\frac{i}{k}\right) \text { to } I \times I, \\
& u_{i+1}(x, y)=u_{i}(x, y) \quad \text { for }(x, y) \in K\left(\frac{i}{k}\right), \\
& u_{i+1}(x, y)=z(x, y)+F_{k}\left(\widetilde{u_{i}}\right)(x, y) \quad \text { for }(x, y) \in K\left(\frac{i+1}{k}\right) \backslash K\left(\frac{i}{k}\right) .
\end{aligned}
$$

Put $u(x, y)=\lim _{i \rightarrow \infty} u_{i}(x, y)$. This convergence is uniform on every compact subset of $I \times I$. Hence in view of the continuity of $F_{k}$, we obtain $u=z+F_{k}(u)$, so $z \in\left(I-F_{k}\right)(C)$.

In view of Theorem 2 the set $T^{-1}(0)$ is an $R_{\delta}$, which completes our proof.

\section{REFERENCES}

[1] A. Ambrosetti, 'Un teorema di esistenza per le equazioni differenziali negli spazi di Banach', Rend. Sem. Math. Univ. Padova 39 (1967), 349-360. 
[2] J. Banaś and K. Goebel, 'Measures of noncompactnes in Banach spaces', in Lecture Notes in Pure and Appl. Math. 60 (Marcel Dekker, New York and Basel, 1980).

[3] D. Bugajewski, 'Weak solutions of integral equations with weakly singular kernel in Banach spaces', Comm. Math. 34 (1994), 49-58.

[4] J.L. Kelley and I. Namioka, Linear topological spaces (Van Nostrand, Princeton, 1963).

[5] D. Ozdarska, 'On the equation $x^{\prime}=g\left(t, x, x^{\prime}\right)$ in Banach spaces', Rad. Mat. 2 (1991), $363-370$.

[6] B. Rzepecki, 'On the existence of solutions of the Darboux problem for the hyperbolic partial differential equations in Banach spaces', Rend. Sem. Math. Univ. Padova 76 (1986), 201-206.

[7] S. Szufla, 'Solution sets on nonlinear equation', Bull. Acad. Polon. Scien. 11 (1973), 971-976.

Faculty of Mathematics and Computer Science

A. Mickiewicz University

Matejki 48/49

60-769 Poznań

Poland

e-mail: dbw@math.amu.edu.pl 